\begin{tabular}{|l|l|}
\hline Asian Journal of Pharmaceutical Research \\
and Development
\end{tabular}

Open $\bigcirc$ Access

Available online at http://ajprd.com/index.php

Research Article

\title{
RECENT INSIGHTS ON PROSPECTS OF CANCER NANOTECHNOLOGY
}

\author{
1Manish Kumar, 2Hemant K. Sharma* \\ ${ }^{1}$ College of Pharmacy, Sri SatyaSai University of Technology and Medical Sciences, Village-Pachama, District- \\ Sehore, Madhya Pradesh-466001, India. \\ ${ }^{2}$ Professor \& Dean, College of Pharmacy, Sri SatyaSai University of Technology and Medical Sciences, Village- \\ Pachama, District- Sehore, Madhya Pradesh-466001, India.
}

\begin{abstract}
Cancer is an extremely multifaceted illness to appreciate, since it entails manifold cellular physiological system. The mainly general cancer treatment is limited to chemotherapy, radiation and surgery. Furthermore, the untimely credit and action of cancer relics a technological block. There is an urgent require to expand novel and originaltechnology thatcould help to define tumor margins, recognize residual tumor cells and micro metastases, and decide whether a tumor has been totally removed or not. Nanotechnology has witnessed significant progress in the past few decades, and its effect is widespread nowadays in every field. Nanoparticles can be modified in numerous ways to prolong circulation, enhance drug localization, increase drug efficacy, and potentially decrease chances of multidrug resistance by the use of nanotechnology. Recently, research in the field of cancer nanotechnology has made remarkable advances. In present study review summarizes the application of various nanotechnology-based approaches towards the diagnostics and therapeutics of cancer.
\end{abstract}

Key-Words: Cancer Nanotechnology, Liposomes, Targeted Delivery, Diagnosis, Nano-medicines.

Article Info: Received:03 Aug,2018; Review Completed:20 Sept,2018; Accepted:05 Oct, 2018; Available online 20 Oct,2018

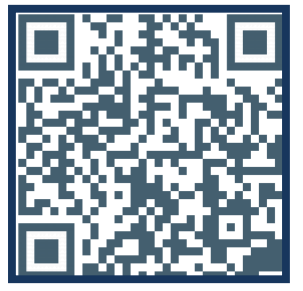

Cite this article as:

Sharma Hemant, Kumar Manish, Recent Insights on Prospects of Cancer Nanotechnology, Asian Journal of Pharmaceutical research and Development.2018;6(5): 46-50

DOI: http://dx.doi.org/10.22270/ajprd.v6i5.413

*Address for Correspondence:

Hemant K. Sharma, Professor \& Dean, College of Pharmacy, Sri SatyaSai University of Technology and Medical Sciences, Village- Pachama, District- Sehore, Madhya Pradesh-466001, India

\section{INTRODUCTION:}

$\mathrm{I}$ n nano-medicine formulation research, developing nanodosage forms (polymeric NPs and Nano capsules, liposomes, solid lipid NPs, phytosomes and Nano emulsionetc) have a number of advantages for delivery system, including enhancement of solubility and bioavailability, protection from toxicity, enhancement of pharmacological activity, enhancement of stability, improving tissue macrophages distribution, sustained delivery, protection from physical and chemical degradation etc ${ }^{1}$.

Scientific advances have significantly improved the basic understanding of biology of cancer. Due to the lack of drug availability, adverse side effects and drug resistance, the conventional therapy failed to achieve proper treatment ${ }^{2}$. During the past few years, nano-medicine has showed considerable progress in improving the cancer treatment, and this review highlights some of the recent advancements in this field of research. After the commencement of nanotechnology in 1959, the field of nano-medicine has developed quickly and we are now successfully approaching solutions to various challenges. The term liposome was described and the drug encapsulated liposome was developed before the 1970s. From there, the advancement of nano-medicine passed through various achievements, starting from gold NP, polymeric NPs, quantum dots, fullerenes etc, to the clinically approved nanomedicines for chemotherapy ${ }^{3}$. The priority of developing nanomaterial for cancer treatment includes ${ }^{4}$ : (i) multifunctionality, (ii) increased potency and multiagency, (iii) increased selectivity for targets, (iv)theranostic potential, (v) altered pharmacokinetics, (vi) controlled synthesis, (vii) controlled agent release and kinetics, (viii) novel properties and interactions, (ix) lack of immunogenicity and (x) enhanced physical stability.

\section{VARIOUS NANOTECHNOLOGY PLATFORMS FOR CANCER THERAPEUTIC}

The most common examples of nanotechnology platforms for cancer therapy include polymeric NPs, 
liposomes, dendrimers, nanoshells, carbon nanotubes, and superparamagnetic NPs. With small size and various structural and physicochemical features, these nanotechnology platforms can enter tumor vasculature through enhanced permeability and retention effect (EPR). The use of cancer specific targeting residues (e.g. antibodies, ligands, and lectins) can also achieve tumor cell targeting.

Nanoshells as the layerbylayer assembly of NPs, polymeric nanoshells (20-60 nm) of di-block copolymers can be made by selfassembly of oppositely charged polymers forming a core/shell structure ${ }^{5}$. The most useful nanoshells are that that have a silica core diameter of 120 $\mathrm{nm}$ with a $10 \mathrm{~nm}$ layer of gold shell, because these strongly absorb near infrared (NIR) light $800 \mathrm{~nm}$ and can create intense heat that is lethal to cells. This NIR light can penetrate several centimeters of human tissue without causing harm, because tissue chromophores do not absorb much energy in the NIR range 1 . The benefit of the nanoshell mediated approach is that the energy can pass through the healthy tissue and leave the neighboring cells intact, while killing only the tumor cells that have been targeted by the nanoshells ${ }^{6}$.

Carbon Nanotubes: - Carbon nanotubes are a distinct molecular form of carbon atoms that was discovered in the late $1980 \mathrm{~s}^{7}$. There has been tremendous enthusiasm over carbon nano-tube applications in many industrial sectors, in part because they have been actively promoted as possessing the advantages of being 100 times stronger than steel with only one-sixth of its weight, and with unusual heat and conductivity properties

Dendrimers:Dendrimers are extensively studied nanocarriers; they are uniformly distributed complex molecules with branched architecture ${ }^{8}$. Dendrites are able to carry hydrophobic as well as hydrophilic drugs due to the presence in them of hydrophobic core and hydrophilic surface. The size, shape and pharmacokinetics of dendrimers depend on the generation number, chemical composition of core and branches as well as surface function group. Chemical modification also can significantly alter the pharmacokinetics and biodistribution of dendrimers. Dendrimers have been used for various applications such as solubility enhancement, photodynamic therapy, drug delivery, bio imaging, cancer treatment and 3D nanoscale core-shell structures ${ }^{9}$. Polyvalent dendrimers interact simultaneously with multiple drug targets. Dendrimers are spherical polymers that are normally less than $5 \mathrm{~nm}$ in diameter. Their key useful feature is the polymer branches that provide vast amounts of surface area into which therapeutic agents and targeting molecules could be attached ${ }^{10,}{ }^{11}$. The prototypical dendrimer starts with an ammonia $\left(\mathrm{NH}_{3}\right)$ core that is reacted with acrylic acid to produce a tri-acid molecule. They synthesized from an ethylenediamine core a $\mathrm{G}_{5}$ poly (amidoamine) dendrimer whose primary amino groups on the surface were first neutralized through partial acetylation to provide enhanced solubility of the dendrimer and prevent nonspecific targeting interactions during delivery. The application of dendrimers is to conjugate different biofunctionalmoieties, such as folic acid, using complementary DNA (cDNA) oligonucleotides to produce clustered molecules, which target cancer cells that over express the high affinity folate receptor. Linked with multiple types of molecules, which bind selectively to cancer cells, chemotherapy agent can be conjugated with antibodies that act as recognition sites to kill cancer cells. Targeted delivery of small molecular drugs, proteins/peptides and genes can be obtained through this process $^{12}$.

Magneto-fluorescent NPs. These NPs are usually magnetic and fluorescent applied to in vivo imaging rapid screening ${ }^{13}$ in hermotherapy and locoregional delivery of chemotherapeutic agents in cancer treatment ${ }^{14}$.

Ceramic NPs. These materials can be synthesized readily at ambient temperatures with the desired size, shape and porosity and used as drug delivery system for photodynamic cancer therapy ${ }^{15}$. Quantum dots. Quantum dots (QDs) are nanometerscale semiconductor crystals composed of groups II-VI or III-V elements, and are defined as particles with physical dimensions smaller than the exciton Bohr radius ${ }^{16}$. Quantum dots are frequently referred to as nanocrystals in the lay press, although the term nano crystal is not restricted to quantum dots. They range from 2 to $10 \mathrm{~nm}$ in diameter and are made of semiconductors and they are currently being used as probes for high resolution molecular imaging of cellular components and for tracking a cell's activities and movements inside the body. Because semiconductors are poisonous heavy metals, toxicity is a huge obstacle to clinical application of quantum dots for humans. Currently, their application is restricted to in vitro or animal studies, and researchers are actively trying to develop different ways to coat them so that they would be safe for use in people ${ }^{17}$.

Superparamagnetic NPs. Superparamagnetic NPs refer to iron oxide particles or magnetite $\left(\mathrm{Fe}_{3} \mathrm{O}_{4}\right)$ particles that are less than $10 \mathrm{~nm}$ in diameter. They have been around for years as contrasting agents for magnetic resonance imaging (MRI). Many groups have explored the use of magnetic fields to localize magnetic NPs to targeted sites, a system known as magnetic drug targeting. As with other NPs, these superparamagnetic NPs are getting functionalized so as to permit specific tumor targeting. Such as iron oxide NPs can also be made by coating with aliphatic surfactants or liposomes resulting in magnetoliposomes ${ }^{18}$. Magnetic NPs can be remotely activated using electromagnetic fields, and they can also be used to thermally treat cancers ${ }^{19}$. Most recently, superparamagnetic NPs have been used in clinical thermotherapy of locally recurrent prostate cancer ${ }^{20}$.

\section{PRACTICAL APPLICATIONS OF NP'S IN CANCER THERAPY}

The fate of a drug after administration in vivo is determined by a combination of several processes such as distribution, metabolism and elimination when given intravenously ${ }^{21}$ or absorption, distribution, metabolism and elimination when an extravascular route is used ${ }^{22}$, which depends mainly on the physicochemical properties of the drug and therefore on its chemical structure. NPs loaded with anticancer agents can be used successfully to increase drug concentration in cancer tissues and also act at cellular levels, enhancing antitumor efficacy ${ }^{23}$. They 
can be endocytosed or phagocytosed by cells, with resulting cell internalization of the encapsulated drug. NPs of biodegradable polymers can provide controlled and targeted delivery of the drug with better efficacy and fewer side effects ${ }^{24}$. Lipophilic drugs, which have some solubility either in the polymer matrix or in the oily core of nanocapsules, are more readily incorporated than hydrophilic compounds, although the latter may be adsorbed onto the particle surface.Nanospheres can also be formed from natural macromolecules such as proteins and polysaccharides, from non polar lipids, and from inorganic materials such as metal oxides and silica ${ }^{25}$.

Prostate cancer is the most common cancer in men and is the sixth leading cause of cancer mortality in $\operatorname{men}^{26}$. In human prostate cancer, a multistage process involves progression from small latent carcinomas of low histological grade to high-grade metastatic cancer ${ }^{27}$. To enhance the therapeutic efficacy of anticancer agents in general, polymeric-, misceller- and liposome-based delivery systems conjugated to tumor-specific ligands have been studied ${ }^{28-30}$. NPs conjugated ligand can enhance the therapeutic efficacy of the encapsulated drug and thus could be more effective in promoting tumor regression than the drug dissolved in the cremophor el (CrEL) formulation ${ }^{31}$.

Nanomedicine can be used to design artificial red and white blood cells successfully ${ }^{32}$. Cancer patients are now treated by injecting artificial red blood cells to balance the human body blood level. Artificial antibodies, white and red blood cells and antiviral nanorobots could be considered as successful applications of nanomedicine ${ }^{33}$. Paclitaxel is one of the best antineoplastic drugs found in nature in the past decades ${ }^{34}$. It is effective in treating a wide spectrum of cancers including breast cancer, ovarian cancer, lung cancer, Kaposi's sarcoma ${ }^{35}$, colon cancer, bladder cancer, head and neck cancer, multiple myeloma and melanoma. Its poor solubility in water creates difficulties in clinical administration and can create many serious side effects ${ }^{36}$. NPs of biodegradable polymers can be considered to counteract the side effects and achieve controlled and targeted delivery of the $\operatorname{drug}^{37}$. By optimization of particle size and surface coating, NPs formulation of paclitaxel could improve the efficacy and quality of chemotherapy, making possible personalized chemotherapy, local chemotherapies, sustained chemotherapy, oral chemotherapy, and chemotherapy across the blood-brain barrier ${ }^{38}$, chemotherapy across the microcirculation barrier, and other advancements. The hydrophobic cytotoxic drug camptothecin ${ }^{39}$ incorporated into fluorescent mesoporous silica NPs can be delivered to various cancer cells to induce cell death. This can also be a method to overcome the insolubility problem of many anticancer drugs, which is considered to be one of the major challenges in cancer therapy ${ }^{40}$. NPs are useful delivery vehicles for promising drug candidates that face obstacles for clinical applicability. Sirolimus, an inhibitor of mammalian target of rapamycin has gained attention for targeted anticancer therapy, but its clinical application has been limited by its poor solubility ${ }^{41}$. Polymeric nanoparticle (PNP)-sirolimus was developed as an injectable formulation and has been characterized by transmission electron microscopy and dynamic light scattering. Pharmacokinetic analysis revealed that PNP- sirolimus has prolonged circulation in the blood ${ }^{42}$. PNPsirolimus preserved the in vitro killing effect of free sirolimus against cancer cells, and intravenous administration displayed its potent in vivo anticancer efficacy in xenograft tumor mice.

Table: 1 Nano medicine for anti cancer therapy

\begin{tabular}{lll}
\hline Trade name & Compound & Nanocarrier \\
\hline Abraxane & Paclitaxel & Albumin bound paclitaxel \\
DaunoXome & Daunorubicin & Pegylated Liposome \\
Doxil & Doxorubicin & Pegylated Liposome \\
Bexxar & $\begin{array}{l}\text { Anti-CD20 } \\
\text { conjugated to }\end{array}$ & Radioimmunoconjugate \\
idodine131 & $\begin{array}{l}\text { Anti CD 20 } \\
\text { conjugated to } \\
\text { yittrium-90 }\end{array}$ & Radioimmunoconjugate \\
Zevalin & $\begin{array}{l}\text { Goserelin } \\
\text { Doxorubicin }\end{array}$ & $\begin{array}{l}\text { Acetate Polymer rods } \\
\text { Non-pegylated liposome }\end{array}$ \\
Myoset & $\begin{array}{l}\text { PEG-L- } \\
\text { asparaginase }\end{array}$ & $\begin{array}{l}\text { Polymer-protein } \\
\text { conjugate }\end{array}$ \\
Ontak & $\begin{array}{l}\text { IL 2 fused to } \\
\text { diphtheria toxin }\end{array}$ & $\begin{array}{l}\text { Immuno toxin fusion } \\
\text { protein }\end{array}$ \\
\hline SMANCS & Zinostatin & $\begin{array}{l}\text { Polymer protein } \\
\text { conjugate }\end{array}$ \\
\hline
\end{tabular}

\section{RECENT PROGRESS OF NANO-MEDICINE IN CLINICAL TRIALS OF ANTICANCER DRUGS}

The FDA has approved the first clinical trial in humans of brightly glowing NPs to light up cancer cells to aid in diagnosing and treating cancer. The trial with five melanoma patients at Memorial Sloan-Kettering Cancer Center (MSKCC) will test if the technology is safe and effective in humans. The NPs, called cornell dots for the university that conducted the research, are silica spheres less than 8 nanometers in diameter that enclose several dye molecules. The silica shell, essentially glass, is chemically inert and small enough to pass through the body and out in the urine. For clinical applications, the dots are coated with polyethylene glycol so the body will not recognize them as foreign substances. To make the dots stick to tumor cells, organic molecules that bind to tumor surfaces or specific locations within tumors can be attached to the shell. When exposed to near-infrared light, the dots glow much brighter than the un encapsulated dye, which serves as a beacon to identify the target cells. For the human trials, the dots will be labeled with radioactive iodine, which makes them visible in PET scans to show how many dots are taken up by tumors and where else in the body they go and for how long. The researchers say this technology can show the extent of a tumor's blood vessels, cell death, treatment response, and invasive or metastatic spread to lymph nodes and distant organs Targeted therapeutic NPs that accumulate in tumors while bypassing healthy cells have shown promising results in an ongoing clinical trial. The NPs feature a homing molecule that allows them to specifically attack cancer cells, and are the first such targeted particles to enter human clinical studies. Originally developed by researchers at MIT and Brigham and Women's Hospital in Boston, the particles are 
designed to carry the chemotherapy of drug docetaxel, used to treat lung, prostate and breast cancers, among others. The particles were also shown to be safe and effective: many of the patients' tumors shrank as a result of the treatment, even when they received lower doses than those usually administered ${ }^{43}$.

\section{RECENT DEVELOPMENTS OF NPS BASED ANTICANCER DRUG}

Nanoscale drug devices are currently being developed to deliver anticancer therapeutics specifically to tumors ${ }^{44}$. But most of the existing anticancer agents cannot distinguish between cancerous and healthy cells, leading to toxic actions and side effects. To further improve drug delivery efficiency and cancer specificity, tumortargeting strategies have recently received significant attention ${ }^{45,46}$. NPs and liposomes are the first generation of these devices. Some of them have already reached clinical practice, such as liposomal doxorubicin used to treat specific forms of cancer, or liposomal amphotericin $B$ used to treat fungal infections often associated with aggressive anticancer treatment ${ }^{47}$. Recently, a nanoparticulate formulation of the well-known anticancer compound taxol was submitted as a new treatment for advanced stage breast cancer ${ }^{48}$. One of the most promising tumor targeting approaches is the investigation of $\mathrm{pH}$-sensitive drug delivery systems ${ }^{49}$, as the existing $\mathrm{pH}$ of tumor tissue is generally considered an ideal trigger for the selective release of anticancer drugs ${ }^{50,51}$. Compared to normal tissue $\mathrm{pH} 7.4$, the average extracellular $\mathrm{pH}$ value in tumor tissues is 6.8 , and the $\mathrm{pH}$ values of intracellular components such as endosomes and lysosomes are 4.5-6.5, which is caused by hypoxia in poorly perfused regions due to the high metabolic rate required for tumor growth ${ }^{52}$. The specificity of lower $\mathrm{pH}$ levels within the tumor region can be a strategy for acidsensitive drug delivery at local microenvironments for not only improving the efficacy of chemotherapy, but also for reducing the level of cytotoxicity ${ }^{53}$. Recent studies have highlighted the development of some drug carriers with $\mathrm{pH}$-sensitive, and therefore tumor-selective, drug delivery. Strontium carbonate NPs (SCNs), a novel biodegradable nano-system for the $\mathrm{pH}$ sensitive release of anticancer drugs, were developed via a facile mixed solvent method aimed at creating smart drug delivery in acidic conditions, particularly in tumor environments ${ }^{54}$. Structural characterization of SCNs revealed that the engineered nanocarriers were uniform in size and

\section{REFERENCES}

1. Yang $Y$, Aw J, Chen $K$, et al. Enzyme-responsive multifunctional magnetic nanoparticles for tumor intracellular drug delivery and imaging. Chem Asian J. 2011; 6(6):1381-1389.

2. Gupta S, Andresen H, Stevens MM. Single-step kinase inhibitor screening using a peptide-modified gold nanoparticle platform. ChemCommun (Camb). 2011; 47(8):2249-2251.

3. Bharali DJ, Khalil M, Gurbuz M, Simone TM, Mousa SA. Nanoparticles and cancer therapy: a concise review with emphasis on dendrimers. Int J Nanomedicine. 2009; 4:1-7.

4. Baker JR. Dendrimer-based nanoparticles for cancer therapy. Hematology Am SocHematolEduc Program. 2009; 2009(1):708719.

5. Svenson S, Tomalia DA. Dendrimers in biomedical applications reflections on the field. Adv Drug Deliv Rev. 2005;57(15):21062129.

6. Tekade RK, Kumar PV, Jain NK. Dendrimers in oncology: an expanding horizon. Chem Rev. 2009;109(1):49-87. presented a dumbbell-shaped morphology with a dense mass of a scale-like spine coating, which could serve as the storage structure for hydrophobic drugs. Chosen as a model anticancer agent, etoposide was effectively loaded into SCNs based on a simultaneous process that allowed for the formation of the nanocarriers and for drug storage to be accomplished in a single step. The etoposide loaded SCNs (ESCNs) possess both a high loading capacity and efficient encapsulation. It was found that the cumulative release of etoposide from ESCNs is acid-dependent, and that the release rate is slow at a $\mathrm{pH}$ of 7.4 ; this rate increases significantly at low $\mathrm{pH}$ levels 5.8 and 3.0. Meanwhile, it was also found that the blank SCNs were almost nontoxic to normal cells, and ESCN systems were evidently more potent in antitumor activity compared with free etoposide, as confirmed by a cytotoxicity test using an MTT assay and an apoptosis test with fluorescence-activated cell sorter (FACS) analysis. These findings suggest that SCNs hold tremendous promise in the areas of controlled drug delivery and targeted cancer therapy ${ }^{55}$.

\section{CONCLUSION}

Nanotechnology is a speedily rising fields that has known novel expect in the action of numerous diseases. Early revealing and behavior of cancer residue a challenge to the scientific community. Moreover, different strategies have been explored in recent years for cancer detection and therapy. Application of nanotechnology in cancer treatment seems to solve these limitations, which has given new hope to humanity. Specific targeting of cancer cells was also the major challenge faced by conventional therapeutic approaches of cancer treatment. Recently, various NP-based drug-delivery systems such as liposomes, dendrimers, diamondoids, QDs, viral NPs, and CNTs have shown encouraging results in cancer therapy. Properties like prolonged existence in systemic circulation, enhanced drug localization, and their efficacy make the NP-based model an excellent one. One of the major challenges in cancer treatment, ie, MDR, can also be overcome by these NP formulations. Inthe light of our review, we expect that in future, different NP formulations would serve as "Trojan horses" in the field of cancer diagnostics and its treatment. Hereby, we find it pertinent to highlight that toxicity and immune-system induction should be given due consideration before finalizing the use of any NP formulation for diagnostic and treatment purposes.

7. Menjoge AR, Kannan RM, Tomalia DA. Dendrimer-based drug and imaging conjugates: design considerations for nanomedical applications. Drug Discov Today. 2010;15(5-6):171-185.

8. Tomalia DA, Reyna LA, Svenson S. Dendrimers as multi-purpose nanodevices for oncology drug delivery and diagnostic imaging. BiochemSoc Trans. 2007; 35(Pt 1):61-67.

9. Padilla De Jesús OL, Ihre HR, Gagne L, Fréchet JMJ, Szoka FC Jr. Polyester dendritic systems for drug delivery applications: in vitro and in vivo evaluation. Bioconjug Chem. 2002; 13(3):453-461.

10. Lai PS, Lou PJ, Peng CL, et al. Doxorubicin delivery by polyamidoaminedendrimer conjugation and photochemical internalization for cancer therapy. J Control Release. 2007; 122(1):39-46

11. Kirkpatrick GJ, Plumb JA, Sutcliffe OB, Flint DJ, Wheate NJ. Evaluation of anionic half generation 3.5-6.5 poly(amidoamine) dendrimers as delivery vehicles for the active component of the anticancer drug cisplatin. J InorgBiochem. 2011; 105(9):11151122

12. Gillies ER, Fréchet JMJ. Dendrimers and dendritic polymers in drug delivery. Drug Discov Today. 2005; 10(1):35-43. 
13. Malik N, Evagorou EG, Duncan R. Dendrimer-platinate: a novel approach to cancer chemotherapy. Anticancer Drugs. 1999; 10(8):767-776.

14. Lee CC, Gillies ER, Fox ME, et al. A single dose of doxorubicinfunctionalized bow-tie dendrimer cures mice bearing $C-26$ colon carcinomas. ProcNatlAcadSci U S A. 2006; 103(45):16649-16654.

15. Maeda H, Wu J, Sawa T, Matsumura Y, Hori K. Tumor vascular permeability and the EPR effect in macromolecular therapeutics: a review. J Control Release. 2000;65(1-2):271-284.

16. Han G, Ghosh P, Rotello VM. Multi-functional gold nanoparticles for drug delivery. Adv Exp Med Biol. 2007; 620:48-56.

17. Chen J, McLellan JM, Siekkinen A, Xiong Y, Li ZY, Xia Y. Facile synthesis of gold-silver nanocages with controllable pores on the surface. J Am Chem Soc. 2006; 128(46):14776-14777.

18. Alkilany AM, Murphy CJ. Toxicity and cellular uptake of gold nanoparticles: what we have learned so far? J Nanopart Res. 2010; 12(7):2313-2333.

19. Kim B, Han G, Toley BJ, Kim CK, Rotello VM, Forbes NS. Tuning payload delivery in tumour cylindroids using gold nanoparticles. Nat Nanotechnol. 2010; 5(6):465-472.

20. Brown SD, Nativo P, Smith JA, et al. Gold nanoparticles for the improved anticancer drug delivery of the active component of oxaliplatin. J Am Chem Soc. 2010;132(13):4678-4684.

21. Khullar P, Singh V, Mahal A, et al. Bovine serum albumin bioconjugated gold nanoparticles: synthesis, hemolysis, and cytotoxicity toward cancer cell lines. J PhysChem C. 2012; 116(15):8834-8843.

22. Joshi P, Chakraborty S, Dey S, et al. Binding of chloroquineconjugated gold nanoparticles with bovine serum albumin. J Colloid Interface Sci. 2011; 355(2):402-409.

23. Smith AM, Dave S, Nie S, True L, Gao X. Multicolor quantum dots for molecular diagnostics of cancer. Expert Rev MolDiagn. 2006;6(2):231-244.

24. Gao X, Dave SR. Quantum dots for cancer molecular imaging. Adv Exp Med Biol. 2007; 620:57-73.

25. Үи $Y, X u$ L, Chen J, et al. Hydrothermal synthesis of GSH-TGA cocapped CdTe quantum dots and their application in labeling colorectal cancer cells. Colloids Surf B Biointerfaces. 2012, 95:247-253.

26. Shen JM, Tang WJ, Zhang XL, Chen T, Zhang HX. A novel carboxymethyl chitosan-based folate/Fe3O4/CdTe nanoparticle for targeted drug delivery and cell imaging. CarbohydrPolym. 2012; 88(1):239-249.

27. Li JM, Wang YY, Zhao MX, et al. Multifunctional QD-based codelivery of siRNA and doxorubicin to HeLa cells for reversal of multidrug resistance and real-time tracking. Biomaterials. 2012; 33(9):2780-2790.

28. Wang $Y$, Zhang $Y, D u Z, W u M$, Zhang $G$. Detection of micrometastases in lung cancer with magnetic nanoparticles and quantum dots. Int J Nanomedicine. 2012; 7:2315-2324.

29. Uner M, Yener G. Importance of solid lipid nanoparticles (SLN) in various administration routes and future perspectives. Int $J$ Nanomedicine. 2007; 2(3):289-300.

30. Wang S, Chen T, Chen R, Hu Y, Chen M, Wang Y. Emodin loaded solid lipid nanoparticles: preparation, characterization and antitumor activity studies. Int J Pharm. 2012; 430(1-2):238-246.

31. Shi SJ, Zhong ZR, Liu J, Zhang ZR, Sun X, Gong T. Solid lipid nanoparticles loaded with anti-microRNA oligonucleotides (AMOs) for suppression of microRNA-21 functions in human lung cancer cells. Pharm Res. 2012; 29(1):97-109.

32. Brewer E, Coleman J, Lowman A. Emerging technologies of polymeric nanoparticles in cancer drug delivery. J Nanomater. 2011; 2011:1-10.

33. Rao JP, Geckeler KE. Polymer nanoparticles: preparation techniques and size-control parameters. ProgPolym Sci. 2011; 36(7):887-913.
34. vanVlerken LE, Amiji MM. Multi-functional polymeric nanoparticles for tumour-targeted drug delivery. Expert Opin Drug Deliv. 2006; 3(2):205-216

35. Owens DE 3rd, Peppas NA. Opsonization, biodistribution, and pharmacokinetics of polymeric nanoparticles. Int $J$ Pharm. 2006;307(1):93-102.

36. Hu S, Zhang Y. Endostar-loaded PEG-PLGA nanoparticles: in vitro and in vivo evaluation. Int J Nanomedicine. 2010;5:1039-1048.

37. Woo HN, Chung HK, Ju EJ, et al. Preclinical evaluation of injectable sirolimus formulated with polymeric nanoparticle for cancer therapy. Int J Nanomedicine. 2012;7:2197-2208.

38. Aravind A, Jeyamohan P, Nair R, et al. AS1411 aptamer tagged PLGA-lecithin-PEG nanoparticles for tumor cell targeting and drug delivery. BiotechnolBioeng. Epub May 21, 2012.

39. Guo J, Gao X, Su L, et al. Aptamer-functionalized PEG-PLGA nanoparticles for enhanced anti-glioma drug delivery. Biomaterials. 2011;32(31):8010-8020.

40. Dhar S, Gu FX, Langer R, Farokhzad OC, Lippard SJ. Targeted delivery of cisplatin to prostate cancer cells by aptamer functionalized Pt(IV) prodrug-PLGA-PEG nanoparticles. ProcNatlAcadSci U S A. 2008;105(45):17356-17361.

41. Chen J, Li S, Shen Q, He H, Zhang Y. Enhanced cellular uptake of folic acid-conjugated PLGA-PEG nanoparticles loaded with vincristine sulfate in human breast cancer. Drug DevInd Pharm. 2011;37(11):1339-1346.

42. Cho H, Bae J, Garripelli VK, Anderson JM, Jun HW, Jo S. Redoxsensitive polymeric nanoparticles for drug delivery. ChemCommun (Camb). 2012.

43. Prajapati BG. Nanoparticles as platforms for targeted drug delivery system in cancer therapy. Internet J Nanotechnol. 2009; 3(1):1-8.

44. Stern JM, Cadeddu JA. Emerging use of nanoparticles for the therapeutic ablation of urologic malignancies. UrolOncol. 2008; 26(1):93-96.

45. Milleron RS, Bratton SB. 'Heated' debates in apoptosis. Cell Mol Life Sci. 2007; 64(18):2329-2333.

46. Cherukuri P, Glazer ES, Curley SA. Targeted hyperthermia using metal nanoparticles. Adv Drug Deliv Rev. 2010; 62(3):339-345.

47. Ellis LM, Curley SA, Tanabe KK. Radiofrequency Ablation for Cancer: Current Indications, Techniques, and Outcomes. New York: Springer-Verlag; 2004.

48. Mody VV, Siwale R, Singh A, Mody HR. Introduction to metallic nanoparticles. J Pharm Bioallied Sci. 2010;2(4):282-289.

49. Samanta B, Yan H, Fischer NO, Shi J, Jerry DJ, Rotello VM. Protein-passivated $\mathrm{Fe} 3 \mathrm{O} 4$ nanoparticles: low toxicity and rapid heating for thermal therapy. J Mater Chem. 2008; 18(11):12041208.

50. Kam NWS, Liu Z, Dai H. Functionalization of carbon nanotubes via cleavable disulfide bonds for efficient intracellular delivery of siRNA and potent gene silencing. J Am Chem Soc. 2005; 127(36): 12492-12493.

51. Gannon CJ, Cherukuri P, Yakobson BI, et al. Carbon nanotubeenhanced thermal destruction of cancer cells in a noninvasive radiofrequency field. Cancer. 2007; 110(12):2654-2665.

52. Samia ACS, Chen X, Burda C. Semiconductor quantum dots for photodynamic therapy. J Am Chem Soc. 2003; 125(51):1573615737.

53. Bakalova R, Ohba H, Zhelev Z, Ishikawa M, Baba Y. Quantum dots as photosensitizers? Nat Biotechnol. 2004; 22(11):1360-1361.

54. Letfullin RR, Iversen CB, George TF. Modeling nanophotothermal therapy: kinetics of thermal ablation of healthy and cancerous cell organelles and gold nanoparticles. Nanomedicine. 2011; 7(2):137145.

55. Misra R. and Sahoo, S.K. Intracellular trafficking of nuclearlocalization signal conjugated nanoparticles for cancertherapy. Eur. J. of Pharm. Sci. 2010; 39:152-163. 